\title{
Investigación formativa en la formación inicial docente
}

\section{Formative research in initial teacher training}

\author{
Giancarlo Fernando Cabrales Lara ${ }^{1 \mathrm{a}}$ \\ Universidad César Vallejo, Trujillo, Perú1
}

Orcid ID: https://orcid.org/0000-0003-1284-6479¹

\section{Resumen}

La investigación formativa (IF) ha despertado gran interés en la comunidad académica porque usa al método científico como medio para que el docente en formación asuma la investigación como parte de su proceso formativo que les permita reflexionar sobre su práctica pedagógica, ensayar alternativas de solución en el campo educativo y tomar decisiones pedagógicas en base a evidencias mejorando su quehacer educativo, tal como lo sostiene López et al. Pero en la actualidad no se ha podido asociar la IF con la dimensión pedagógica, y esto por el vacío definitorio que no la diferencia de la investigación propiamente dicha generando confusión en los docentes formadores. En ese sentido, el presente artículo de revisión sistemática tiene como objetivo conocer las percepciones sobre la cual se está desarrollando la investigación formativa en los institutos y escuelas pedagógicas, y para esto se analizaron 42 artículos como resultado del análisis crítico a través del instrumento LcotAC. Las percepciones encontradas sobre IF muestran que no están alineadas con lo pedagógico, sino al cumplimiento de políticas educativas y a la formación de investigadores. El presente estudio pudo notar también algunas definiciones que proponen los autores sobre IF orientadas a la producción de conocimiento menos estricta y aquella que considera los objetivos de las asignaturas, entre los que destaca Sánchez-Carlessi. Finalmente, este estudio propone una definición integral que contribuirá a identificar la naturaleza y esencia de la IF.

Palabras claves: Investigación formativa, Formación inicial docente, percepciones, definiciones, método científico, pedagogía, educación.

\footnotetext{
Abstract

${ }^{\mathrm{a} C}$ Correspondencia al autor:

E-mail: cabralesg257@gmail.com
}

Formative research (FI) has aroused great interest in the academic community because it uses the scientific method as a means for the teacher in training to assume research as part of their 
training process that allows them to reflect on their pedagogical practice, test alternative solutions in the educational field and make pedagogical decisions based on evidence, improving their educational work, as stated by López et al. But at present it has not been possible to associate FI with the pedagogical dimension, and this due to the defining void that does not differentiate it from the research itself, generating confusion in the teacher-trainers. In this sense, the present systematic review article aims to know the perceptions on which formative research is being developed in pedagogical institutes and schools, and for this, 42 articles were analyzed as a result of critical analysis through the LcotAC instrument. The perceptions found about FI show that they are not aligned with the pedagogical, but with the fulfillment of educational policies and the training of researchers. The present study could also note some definitions proposed by the authors about FI oriented to the production of less strict knowledge and one that considers the objectives of the subjects, among which Sánchez-Carlessi stands out. Finally, this study proposes a comprehensive definition that will help to identify the nature and essence of FI.

Keywords: Formative research, initial teacher training, perceptions, definitions, scientific method, pedagogy, education.

\section{Introducción}

La formación de docentes en investigación ha despertado un gran interés por su importancia que tiene en la mejora de la práctica pedagógica (Mendoza et al., 2020). En esa línea, la Dirección de Formación Inicial Docente del Ministerio de Educación del Perú (DIFOID, 2019), propone articular la práctica pedagógica con la investigación bajo un enfoque formativo. No obstante, los estudiantes ven a la investigación con fines de titulación (GarcíaGutiérrez \& Aznar-Díaz, 2019; Velázquez et al., 2019; Mesa et al., 2020; Colán-Hernández, 2020; Rebolledo-Rebolledo, 2020) y los docentes la ven con fines científicos (Turpo-Gebera et al., 2020). Asimismo, Venegas et al., (2019) concluyen que tanto docentes como estudiantes ven a la investigación formativa como un proceso para gestionar conocimiento.

Por su parte, Sánchez-Carlessi (2017) nos dice que la investigación puede darse bajo dos enfoques: la formal, relacionado con el cumplimiento estricto de las líneas y políticas de investigación institucionales, y la formativa, vista como una investigación de carácter exploratorio que puede realizar el docente y sus estudiantes en el aula. Otros como Tapia et al. (2017); Rebolledo-Rebolledo (2020) hablan de una investigación para el saber disciplinar, relacionado con la investigación científica; y otra para el saber pedagógico, relacionado con lo formativo. Por otro lado, Venegas et al. (2019), distinguen una investigación educativa caracterizada por la producción de conocimiento; y una formativa asociada al diseño curricular. Asimismo, Mas y Vásquez (2019) mencionan dos modelos: la formativa, orientada a lo académico y la investigación en sentido estricto, relacionado con la comunidad científica. 
Las percepciones que se encontraron en el presente estudio de revisión se han agrupado según sus propósitos en dos modelos: como requisito de normas y políticas educativas (GarcíaGutiérrez \& Aznar-Díaz, 2019; Rebolledo-Rebolledo, 2020) con especial atención en la acreditación institucional (Alvitres et al.,2016), y como formación de investigadores (Castro, 2017; Ruano et al., 2017; Ureña, 2018; Vera et al., 2018; Claudio, 2018; Turpo-Gebera et al., 2019; Aranda, 2019; Lizarzauru et al, 2019; Campos, 2020; Iriarte-Pupo, 2020). En el primer modelo se puede notar que los institutos y escuelas pedagógicas priorizan los objetivos institucionales sobre los pedagógicos, esto con miras de lograr la acreditación o licenciamiento institucional. En el segundo modelo se puede observar a la investigación formativa como medio para formar investigadores; sin embargo, López et al. (2018) afirman que la finalidad es lo pedagógico teniendo como medio al método científico.

La investigación formativa tiene su impacto enriquecedor e innovador sobre el quehacer pedagógico, tanto para el docente formador y el futuro docente (Sánchez-Carlessi, 2017). En educación superior la investigación formativa es sinónimo de calidad (Velandia-Mesa et al., 2017), cuyos resultados se observan en el desarrollo socioeconómico de un país (Hernández et al., 2020) y en la transformación de la realidad educativa (Pérez, 2016; Borjas et al., 2016; Aguilar et al., 2018; Urbina et al., 2020).

En consonancia con lo anterior, existen dos tipos de investigaciones: la formal y la formativa; sin embargo, los docentes formadores confunden la naturaleza de ambas investigaciones asumiendo la investigación formativa desde el enfoque rígido de la investigación. Esta realidad también la podemos encontrar en las investigaciones de Ureña (2018); DIFOID (2019); Turpo-Gebera et al. (2020); Campos (2020); Iriarte-Pupo (2020) donde se mencionan que se está perdiendo la esencia de la acción formativa en torno al perfil de egreso de la formación inicial docente. Visto lo anterior, la pregunta de investigación que dinamizó el presente artículo es ¿Cuáles son las percepciones que se tiene sobre la investigación formativa en la formación inicial docente? Esto con el propósito de conocer las percepciones sobre la investigación formativa que manejan los docentes formadores (Turpo-Gebera et al., 2020) y finalmente ofrecer a la comunidad académica una definición que integre fundamentos didácticos, pedagógicos y curriculares de la investigación formativa por ser de gran importancia (López-De Parra, et al., 2017).

\section{Método}

El presente es un estudio de revisión sobre la investigación formativa que parte de una pregunta bien definida y estructurada que busca consultar datos de fuentes de información 
secundaria para responder la pregunta de investigación y luego analizar y sistematizar la información bajo un método ordenado y reproducible (Torres-Fonseca y López-Hernández, 2014).

Se establece una estrategia de búsqueda, donde se incluyen artículos que tienen en el título o resumen con las siguientes palabras claves: investigación formativa, formación inicial docente, percepciones, definiciones; en inglés: formative research, initial teacher training, perceptions, definitions, teniendo en cuenta que la muestra sea de formación inicial de docentes, excluyendo otras carreras profesionales de pregrado, así como las investigaciones para la evaluación formativa. Seguido se localizó y se seleccionó los estudios relevantes con base en los criterios de selección de documentos, luego se extrajo y se recopilaron los datos de los estudios secundarios, asimismo se organizó la información, se analizó y se presentan los resultados, posteriormente se interpretaron los resultados y finalmente se procedió a redactar el artículo.

\section{Instrumento}

Se utilizó una lista de cotejo para el análisis crítico de artículos (LcotAC) adaptado del Programa de Habilidades en Lectura Crítica Español (CASPe) referenciado en Osorio y Cárdenas (2017) y en Salazar y Gastélum (2020), que permitió mediante criterios establecidos valorar el esfuerzo de los autores por referenciar aquellos estudios más actuales y pertinentes en sus investigaciones, así como el proceso coherente de su metodología.

\section{Procedimiento}

Definido el objetivo del presente estudio de revisión (conocer las percepciones sobre la cual se está desarrollando la investigación formativa en los institutos y escuelas pedagógicas), se procedió a establecer los criterios de búsqueda tales como: artículos publicados entre el año 2016 al 2020, la unidad de análisis consistió en estudiantes de pregrado de formación inicial docente, el idioma de publicación en español e inglés (para inglés se usó términos como and, or, not y combinaciones). Las bases de datos consultadas fueron Scopus, EBSCO, Dialnet, DOAJ, SciELO, en estos se aplicó filtros a través de sus tesauros: con en el área temática se eligió las ciencias sociales y educación, con el tipo de documento artículos y libros. Se excluyeron algunas bases de datos que sus tesauros no permitían realizar combinaciones de las palabras claves y otras como WoS que se debía realizar un pago económico por las publicaciones de interés. 
Para la estrategia de selección se dio lectura a los títulos y resúmenes, eligiendo aquellos que se relacionaban con la investigación formativa y palabras claves. De esta selección se aplicó una lectura completa a través de la técnica de lectura veloz seleccionando aquellos que sus muestras coincidían con estudiantes en formación inicial docente. Finalmente, a estos artículos se le aplicó el instrumento LcotAC que es una lista de cotejo para el análisis crítico, seleccionando 42 de estos. El instrumento fue elaborado teniendo en cuenta indicadores que permitan evidenciar la pregunta de investigación, los objetivos y la muestra, así como la relación de estos con el diseño metodológico propuesto (González, 2017). Para la estrategia de aplicación se precisaron puntuaciones a los indicadores (Abad et al, 2003), 1 punto si se evidencia el indicador y 0 puntos si no se evidencia tal como se muestra en la tabla 1.

Tabla 1

Lista de cotejo para el análisis crítico de artículos (LcotAC)

\begin{tabular}{|c|c|c|c|}
\hline Número & Indicadores & $\begin{array}{c}\text { Sí } \\
1\end{array}$ & $\frac{\text { No }}{0}$ \\
\hline 1 & Evidencia la pregunta de investigación & & \\
\hline 2 & Evidencia los objetivos de la investigación & & \\
\hline 3 & $\begin{array}{l}\text { Los objetivos están en relación con la pregunta de } \\
\text { investigación }\end{array}$ & & \\
\hline 4 & Se precisa la muestra intervenida & & \\
\hline 5 & $\begin{array}{l}\text { El tipo de artículo se orienta con los objetivos de la } \\
\text { investigación }\end{array}$ & & \\
\hline 6 & $\begin{array}{l}\text { La metodología aplicada es apropiada para los } \\
\text { objetivos de la investigación }\end{array}$ & & \\
\hline 7 & $\begin{array}{l}\text { Los resultados están en función con los objetivos } \\
\text { de la investigación }\end{array}$ & & \\
\hline 8 & Se precisa la discusión de los resultados & & \\
\hline 9 & $\begin{array}{l}\text { Las conclusiones están en función con los } \\
\text { objetivos de la investigación }\end{array}$ & & \\
\hline 10 & Se evidencia una bibliografía actualizada & & \\
\hline 11 & $\begin{array}{l}\text { Se evidencian en la bibliografía las investigaciones } \\
\text { más pertinentes }\end{array}$ & & \\
\hline 12 & $\begin{array}{l}\text { Se evidencia que las recomendaciones están } \\
\text { alineadas con los hallazgos del estudio }\end{array}$ & & \\
\hline
\end{tabular}

Nota. La valoración de los artículos usando la tabla se determina por puntuaciones (menores a 7 puntos no se considera el artículo y mayores o iguales a 7 puntos se considera el artículo). Después de la selección final de artículos se procedió a organizar la información a través de tablas teniendo en cuenta varios criterios: el número de artículos revisados por fases de revisión, la frecuencia de artículos según el año, idioma y país, las principales definiciones 
de investigación formativa, así como sus percepciones (Turpo-Gebera et al., 2020). Luego se procedió a la discusión de los resultados en función de los autores más destacados estableciendo las condiciones de la realidad de la investigación formativa en la formación inicial docente. Finalmente, y en consecuencia de lo revisado se propuso una definición integral de investigación formativa.

\section{Resultados}

Tabla 2

Número de artículos revisados

\begin{tabular}{lc}
\multicolumn{1}{c}{ Artículos } & Número \\
\hline Total encontrados & 633 \\
Total seleccionados al leer resúmenes y & 82 \\
títulos & 61 \\
Total seleccionados al leerlos completos & 42 \\
Total seleccionados después de la lectura \\
crítica $^{\text {a }}$
\end{tabular}

${ }^{\mathrm{a}}$ Se aplicó el instrumento LcotAC.

En la tabla 2, se pueden observar los resultados de la búsqueda de artículos. Aplicando los filtros y palabras claves, se encontraron 633 artículos, de los cuales se seleccionaron 82, porque sus títulos y resúmenes se relacionaban con la variable de estudio y la muestra; de los mismos se seleccionaron 61 artículos por lectura completa, porque sus contenidos se relacionaban con los propósitos de la presente investigación; de los restantes (61) se excluyeron 19 artículos, porque no cumplieron con los criterios del instrumento LcotAC, seleccionándose 42 artículos.

Tabla 3

Distribución de la frecuencia de artículos teniendo en cuenta año, idioma y país

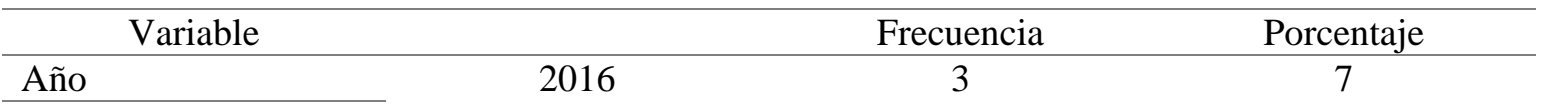




\begin{tabular}{lccc}
\hline & 2017 & 9 & 22 \\
& 2018 & 8 & 19 \\
& 2019 & 11 & 26 \\
\multirow{4}{*}{ Idioma } & 2020 & 11 & 26 \\
\multirow{3}{*}{ Por País } & Inglés & 6 & 14 \\
& Español & 36 & 86 \\
& Colombia & 14 & 33 \\
& Perú & 14 & 33 \\
& Ecuador & 7 & 17 \\
& España & 5 & 12 \\
\hline
\end{tabular}

${ }^{a}$ Un artículo por país.

En la tabla 3, podemos observar que el año con mayores números de publicaciones es el 2019, del mismo modo el idioma de mayor publicación es el español, y el país con mayor estudio sobre la variable de esta investigación es Colombia, seguido de Perú.

Tabla 4

Principales definiciones sobre investigación formativa

\begin{tabular}{|c|c|c|c|}
\hline Autores & Definiciones & Autores & Definiciones \\
\hline $\begin{array}{l}\text { Sánchez-Carlessi } \\
\text { (2017). }\end{array}$ & $\begin{array}{l}\text { Estrategia didáctica } \\
\text { que } \\
\text { considera los } \\
\text { objetivos y } \\
\text { contenidos de las } \\
\text { asignaturas. }\end{array}$ & $\begin{array}{l}\text { (Bernal et al., 2018, p. } \\
\text { 102). }\end{array}$ & $\begin{array}{l}\text { "Generación de } \\
\text { conocimiento menos } \\
\text { estricta" }\end{array}$ \\
\hline $\begin{array}{l}\text { López-de Parra et al. } \\
\text { (2017); Tapia et al. } \\
\text { (2017); Companioni } \\
\text { (2019); Sabariego et } \\
\text { al. (2020) }\end{array}$ & $\begin{array}{l}\text { Herramienta } \\
\text { pedagógica con } \\
\text { influencia en las } \\
\text { competencias } \\
\text { investigativas. }\end{array}$ & López et al. (2018). & $\begin{array}{l}\text { Proceso de } \\
\text { aprendizaje por } \\
\text { métodos de } \\
\text { investigación }\end{array}$ \\
\hline Rodríguez, 2019, p.17 & $\begin{array}{l}\text { "Formación en } \\
\text { y para la } \\
\text { investigación" }\end{array}$ & $\begin{array}{l}\text { Álvarez et al. (2017); } \\
\text { Ramírez et al. (2017); } \\
\text { Norton (2018); Conde } \\
\text { (2018); Venegas et al. } \\
\text { (2019); Mas y } \\
\text { Vásquez (2019); } \\
\text { Turpo-Gebera (2020). }\end{array}$ & Investigación acción \\
\hline
\end{tabular}

Nota. Las definiciones en el presente estudio se han considerado como explicaciones claras y exactas de las características generales y diferenciales de la investigación formativa.

De la tabla 4, podemos notar las principales definiciones sobre investigación formativa en 15 artículos de los 42 seleccionados, de los cuales observamos 2 enfoques: la orientada a la investigación y la orientada a la formación. 
Tabla 5

Principales percepciones encontradas sobre investigación formativa

\begin{tabular}{|c|c|c|c|}
\hline Autores & $\begin{array}{c}\text { Percepciones } \\
\text { Encontradas }\end{array}$ & Autores & Percepciones \\
\hline
\end{tabular}

Ureña, (2018); Vera

et al. (2018); Aranda

(2019); Gebera et al

(2019); Iriarte-Pupo

Formación de

investigadores

(2020); Campos

(2020).

García-Gutiérrez y

Aznar-Díaz (2019);

Rebolledo-Rebolledo (2020).
Castro (2017); Ruano et al. (2017); Claudio (2018); Lizarzauru et Actitud favorable para investigar al. (2019).

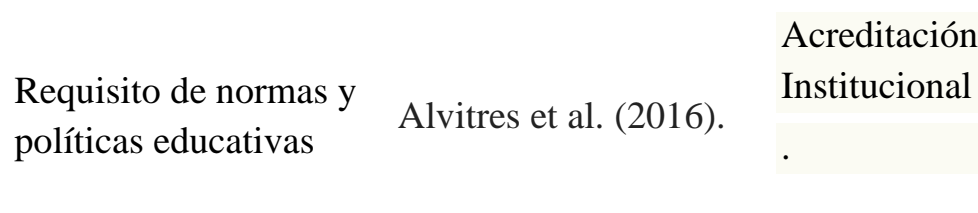

Nota. Las percepciones en el presente estudio se han considerado como una idea, creencia u opinión que se limita al simple conocimiento de la investigación formativa.

En la tabla 5, podemos observar las principales percepciones encontradas por los autores sobre investigación formativa en 12 artículos de los 42 seleccionados, de los cuales observamos 2 modelos: como requisito de normas y políticas educativas (Alvitres et al., 2016; GarcíaGutiérrez, \& Aznar-Díaz, 2019; Rebolledo-Rebolledo, 2020); y como formación de investigadores (Castro, 2017; Ruano et al., 2017; Ureña, 2018; Vera et al., 2018; Claudio, 2018; Aranda, 2019; Lizarzauru et al, 2019; Campos, 2020; Gebera et al., 2020; Iriarte-Pupo, 2020).

\section{Discusión}

El presente trabajo ha revisado artículos del 2016 al 2020 que mencionan estudios sobre la investigación formativa en la formación inicial docente; observándose percepciones y distintas definiciones sobre las cuales se enfoca la investigación formativa en los institutos y escuelas pedagógicas. Para la Real Academia Española, la percepción se entiende como una idea, creencia u opinión que se limita al simple conocimiento de algo, en tanto las definiciones son explicaciones claras y exactas de las características generales y diferenciales de algo. En ese sentido, notamos que la realidad de la investigación formativa depende de los supuestos (Turpo-Gebera et al, 2020) o fundamentos que integren la interpretación de la misma.

Las percepciones encontradas se pueden agrupar según sus propósitos en dos modelos: como requisito de normas y políticas educativas con especial atención en la acreditación institucional (Alvitres et al., 2016; García-Gutiérrez, \& Aznar-Díaz, 2019; RebolledoRebolledo, 2020). Estos autores manifiestan que los docentes ven la investigación formativa 
como medio para cumplir los objetivos institucionales basados en el cumplimiento de los estándares nacionales e internacionales y en la especialización del conocimiento, sin embargo, para Sánchez-Carlessi (2017) la IF debe partir de los objetivos pedagógicos de los cursos. Este primer modelo encontrado nos permite darnos cuenta de cómo se está desnaturalizando el verdadero propósito de la investigación formativa en los institutos y escuelas pedagógicas, priorizando el cumplimiento de los objetivos institucionales sobre los pedagógicos.

El segundo modelo de las percepciones encontradas está relacionado con la formación de investigadores, dentro del cual destaca una actitud favorable para investigar, aquí los docentes perciben a la investigación formativa como formación para la investigación con fines de producción de conocimiento, en contraste con esto López et al., (2018) precisan que el fin de la IF no es lo investigativo sino lo pedagógico usando como medio al método científico. En este segundo modelo los docentes formadores usan la investigación con fines científicos, especializando a los estudiantes en enfoques y diseños metodológicos para cumplir con normas y políticas de investigación; es decir su finalidad es lo investigativo, sin embargo, los estudiantes no están asumiendo la misma como parte de su proceso formativo, que les permita reflexionar sobre su práctica pedagógica y ensayar alternativas de solución en el campo educativo.

En cuanto a las definiciones encontradas de investigación formativa podemos notar que se dan bajo dos enfoques: la primera está orientada a la investigación, en este enfoque observamos varias líneas: incluida la de herramienta pedagógica con influencia en las competencias investigativas, la formación en y para la investigación, la generación de conocimiento menos estricta, y la investigación acción. Las mencionadas líneas de este enfoque consideran lo pedagógico, aunque en algunas proporciones, es decir usan lo pedagógico como medio para fines investigativos, sin embargo, se debe usar el método científico como medio para fines pedagógicos (López et al., 2018). El segundo enfoque observado de las principales definiciones está orientado a la formación, aquí tenemos dos líneas: la de estrategia didáctica que considera los objetivos y contenidos de las asignaturas (Sánchez-Carlessi, 2017) y la del proceso de aprendizaje por métodos de investigación (López et al., 2018). Ambas líneas de este enfoque priorizan lo formativo y usan como herramienta a la investigación.

Queda claro que entender la investigación formativa ha generado diversas interpretaciones (Turpo-Gebera et al., 2020), tal situación nos lleva a peguntar ¿Cuál es la finalidad de la investigación formativa? y para ello se debe analizar los significados de sus términos: investigación y formativa. La primera según la Real Academia Española su propósito es aumentar conocimiento, y la segunda tiene la finalidad de formar. Uniendo ambas finalidades 
entendemos a la investigación formativa como la generación de conocimientos con propósitos formativos. En consonancia con lo anterior, se debe consensuar una definición de IF bajo el enfoque formativo desde lo pedagógico donde se precise los elementos que forman parte de su naturaleza y esencia.

El presente estudio de revisión destaca dos aproximaciones definitorias sobre investigación formativa, la de Sánchez-Carlessi (2017) quien la considera como estrategia didáctica que incorpora los objetivos de las asignaturas, y la de López et al. (2018), quienes precisan que el proceso de enseñanza y aprendizaje debe girar en torno al método científico. El primer autor considera a la investigación formativa como estrategia didáctica, pero destacamos también en su definición el aporte de estrategia pedagógica cuando menciona que se deben considerar los objetivos de las asignaturas. Los segundos autores dejan en claro la finalidad y el medio en la IF. Ambos aportes servirán de base para construir y proponer una definición que sirva a la comunidad académica como directriz para su implementación eficaz.

En consonancia con lo anterior, el presente estudio de revisión propone que la investigación formativa se asuma como estrategia didáctica, pedagógica y curricular que favorece el desarrollo de las competencias del perfil de egreso en función de los estándares de formación inicial docente. Esta propuesta presenta tres fundamentos importantes: lo didáctico, porque considera al método científico en el proceso enseñanza aprendizaje; lo pedagógico, porque toma en cuenta los desempeños específicos previstos en los estándares de formación inicial docente; y lo curricular porque articula verticalmente los contenidos de los cursos de un mismo ciclo académico a través de proyectos integradores. Por último, la definición propuesta va a contribuir en los docentes formadores una comprensión integral de la investigación formativa, y como consecuencia de esto los estudiantes podrán desarrollar habilidades investigativas para reflexionar sobre su práctica y tomar decisiones pedagógicas en base a evidencias, mejorando su quehacer educativo.

\section{Limitaciones}

Como limitación al presente estudio se tuvieron que no se realizaron las combinaciones suficientes de las palabras claves de esta investigación a través de los términos booleanos como and, or, not en los buscadores, lo que imposibilitó posiblemente la identificación de más artículos sobre la temática en el idioma inglés; Así mismo en la revisión se propuso que fueran totalmente en artículos, pero no se encontraron muchos artículos sobre este estudio por ser un estudio actual, en ese sentido se incluyeron algunos libros con información importante sobre la 
IF. También se observa que la temporalidad de los artículos buscados (los últimos 5 años), quizás dejó estudios con información relevante que sume a esta investigación.

\section{Recomendaciones}

Se recomienda a los docentes formadores y comunidad académica a considerar en futuras investigaciones a los estudios cuasiexperimentales correlativos con diseño de series temporales con un grupo control para asociar tres propuestas: investigación formativa y estrategia didáctica (usando al al método científico en el proceso enseñanza aprendizaje); investigación formativa y estrategia pedagógica (considerando los desempeños específicos previstos en los estándares de formación inicial docente) e investigación formativa y estrategia curricular (articulando verticalmente los contenidos de los cursos de un mismo ciclo académico a través de proyectos integradores).

\section{Referencias}

Abad-Corpa, E., Monistrol-Ruano, O., Altarribas-Bolsa, E., y Paredes-Sidrach de Cardona, A. (2003). Lectura crítica de la literatura científica. Enfermería clínica, 13 (1), 32-40. https://doi.org/10.1016/S1130-8621(03)73779-6

Aguilar Barreto, A. J., Rojas Flórez, A. M., Nieto Duarte, B. M., Gomezaquira Contreras, D. A., Díaz Camargo, E. A., Orduz Gualdrón, F. S., Zambrano Quintero, H. R., Portilla Carrillo, J. C., Camacho Galvis, J. M., Barbosa Mora, J. A., Salazar Torres, J. P., Mora Laguado, K. Y., Sánchez Zamora, K. J., Fonseca Hernández, K. S., Barrera Rodríguez, L. V., Guarguati-Duarte, L. A., Ramírez Serrano, M. A., Montánchez, M. L., Luna Moncada, M. A., Contreras-Santander, Y. L. (2018). La investigación educativa: reconociendo la escuela para transformar la educación. Colombia: Ediciones Universidad Simón Bolívar. https://bonga.unisimon.edu.co/bitstream/handle/20.500.12442/2275/La\%20investigaci on\%20educativa.pdf? sequence $=1 \&$ isAllowed $=\mathrm{y}$

Álvarez-Castillo, J. L., Martínez-Usarralde, M. J., González-González, H., \& BuenestadoFernández, M. (2017). El aprendizaje-servicio en la formación del profesorado de las universidades españolas. Revista Española de Pedagogía, 75 (267), 199-217. https://doi.org/10.22550/REP75-2-2017-02 
Alvitres, V., Chambergo, A., \& Fupuy, J. (2016). La investigación formativa y la acreditación universitaria $\quad$ peruana. Revista Manglar, $11 \quad$ 3748. http://dx.doi.org/10.17268/manglar.2014.013

Aranda, A. (2019). La investigación formativa y el emprendimiento e innovación en el currículo de carreras universitarias. Revista Universidad Ciencia y Tecnología, 23 (91), 25-32. http://uct.unexpo.edu.ve/index.php/uct/article/viewFile/945/790

Bernal, O., Barrios, I. \& Hernández, J., (2018). Investigación formativa y estricta, experiencias $\begin{array}{llll}\text { significativas. } & \text { Colombia: } & \text { Editorial }\end{array}$ https://www.researchgate.net/publication/341189583_INVESTIGACION_FORMATI VA_PUNTUALIZACION_ACERCA_DE_SUS_CARACTERISTICAS

Borjas, M. P., Martínez-Gómez, A., Osorio-Villegas, M., \& Jaramillo-de Certain, L. (2016). Los colectivos de investigación: una experiencia de investigación formativa en la Licenciatura de Pedagogía Infantil. España: Editorial Universidad del Norte. https://dialnet.unirioja.es/servlet/libro?codigo $=663571$

Campos-Olazabal, P. J. (2020). La importancia de la investigación formativa como estrategia de aprendizaje. Educare et Comunicare: Revista de investigación de la Facultad de Humanidades, 8 (1), 88-94. https://doi.org/10.35383/educare.v8i1.397

Castro-Molinares, S. P. (2017). Diseño y validación de un instrumento para evaluar la actitud hacia la investigación formativa en estudiantes universitarios. Revista Actualidades Pedagógicas, 1 (70), 165-182. https://doi.org/10.19052/ap.3996

Claudio-Pinedo, J. M. (2018). Investigación formativa y actitud científica en estudiantes de la universidad de Huánuco. Desafíos: Revista Científica De Ciencias Sociales y Humanidades, 9 (1), 3-6. https://doi.org/10.37711/desafios.2019.1.1.61

Colán-Hernández, B. A. (2020). La investigación formativa en el contexto de la universidad peruana. Cienciamatria: Revista Interdisciplinaria de Humanidades, Educación, Ciencia y Tecnología, 6 (11), 183-192. https://doi.org/10.35381/cm.v6i11.354

Companioni, O. L. (2019). Escenarios educativos latinoamericanos. Una mirada desde las universidades. Ecuador: Editoriales UTM Universidad Técnica de Manabí. https://dialnet.unirioja.es/servlet/articulo?codigo=7050555

Conde-Carmona, R. J., Padilla-Escorcia, I. A., \& Valbuena-Duarte, S. (2018). Formación en investigación y práctica pedagógica de docentes de matemáticas: una mirada desde los formadores de formadores, maestros en formación inicial y egresados. En Valbuena, Sonia; Vargas, Leonardo; Berrío, Jesús (Eds.), Encuentro de Investigación en 
Educación Matemática. Editorial Universidad del Atlántico. http://funes.uniandes.edu.co/14306/

DIFOID. (2019). Guía para la implementación de los módulos de práctica e investigación. Minedu.

García-Gutiérrez, Z. D. P., \& Aznar-Díaz, I. (2019). O desenvolvimento de competências investigativas, uma alternativa para formar profissionais em pedagogia infantil como docentes de pesquisa. Revista Electrónica Educare, $23 \quad$ (1), 297-318. http://dx.doi.org/10.15359/ree.23-1.15

González-Aguilera, J. C. (2017). Lectura crítica de la literatura científica: un ejercicio necesario para la $\quad$ práctica. Multimed, $19 \quad$ 982-984. http://www.revmultimed.sld.cu/index.php/mtm/article/view/424

Hernández, R. M., Marino-Jiménez, M., Rivero-Forton, Y., \& Sánchez, N. (2020). Research in university students: real needs for the implementation of a formative research program. Revista Academia, 20-21 (3), 154-176. https://doi.org/10.26220/aca.3445

Iriarte-Pupo, A. J. (2020). Fenomenología-hermenéutica de la investigación formativa, hacia el Ubuntu: el sentido de comunidad. Revista Electrónica: Entrevista Académica, 2 (5), 118. https://ideas.repec.org/a/erv/rearea/y2020i530.html

Lizarzauru-Montero, L., Campos-Marín, B., Campos-Lizarzaburu, W. B., \& FrancoLizarzaburu, R. J. (2019). University research system and formative research system in peruvian universities. Revista Magister Science Journal, 2 (2), 57-76. https://magisterpub.com/ojs/index.php/msj/article/view/42

López-de Parra, L., Polanco-Perdomo, V., \& Correa-Cruz, L. (2017). Mirada a las investigaciones sobre formación investigativa en la universidad latinoamericana: estado del arte 2010 a 2017. Revista de Investigación, Desarrollo e Innovación, 8 (1), 77-95. https://doi.org/10.19053/20278306.v8.n1.2017.7371

López-Falcón, A., Ramos-Serpa, G., \& Gómez-Armijos, C. (2018). La investigación formativa: naturaleza y vínculos recíprocos con la investigación generativa en la educación $\begin{array}{llll}\text { superior. Revista } \quad \text { Uniandes } & \text { Episteme, } 5 \quad \text { (1), }\end{array}$ http://45.238.216.13/ojs/index.php/EPISTEME/article/view/1531

Mas-Sandoval, R. K., \& Torres-Vásquez, C. P. (2019). Investigación formativa para articular la docencia y la responsabilidad social. Editorial Psychology Investigation. https://static.s123-cdn.com/uploads/3743669/normal_5f02288be73c8.pdf 
Mendoza-Velazco, D., Abrigo-Córdova, I., Romero-Chávez, J., Cueva-Bravo, F. \& Cejas, M. F. (2020). The formative research of Ecuadorian university teaching staff. Problems of Education in the 21st century, 77 (3), 734-378. http://dx.doi.org/10.2139/ssrn.3639465 Mesa-Angulo, J. G., González-Gil, E., Castellanos, J. A., Vela-González, P. A., MoncadaGuzmán, C. J., Moreno-Castro, J. A., González-González, J. A., Nieto-Bravo, J. A., Pérez-Vargas, J. J., Bonilla-Mora, M. I., Céspedes-Guervara, N. Y., \& Suárez-Alba, R. (2020). Lineamientos y protocolo de investigación formativa. Colombia: Editorial Universidad Santo Tomas. https://repository.usta.edu.co/bitstream/handle/11634/31137/Protocolo\%20Opciones\% 20de\%20grado_Pregrado.pdf?sequence $=1 \&$ is Allowed $=y$

Norton, L. (2018). Action research in teaching and learning: A practical guide to conducting pedagogical research in universities. Teaching Theology \& Religion, 15 (3), 302-303. https://doi.org/10.1111/j.1467-9647.2012.00821.

Osorio, J. E. \& Cárdenas-Niño, L. (2017). Estrés laboral: estudio de revisión. Diversitas: Perspectivas en psicología,13 (1), 81-90. https://doi.org/10.15332/s1794$\underline{9998.2017 .0001 .06}$

Pérez-Manzano, M. (2016). La investigación formativa, una práctica docente con sentido. Revista Boletín Redipe, 5

125-129. https://revista.redipe.org/index.php/1/article/view/56

Ramírez-Zárate, J., Pulido, Á. R., Rodriguez, H. B., \& Fernandez, Y. L. (2017). La práctica pedagógica e investigativa, como espacio vital de la formación inicial docente en Colombia.

Academia. https://www.academia.edu/33645783/La_pr\%C3\%A1ctica_pedag\%C3\%B3gica_e_inv estigativa_como_espacio_vital_de_la_formaci\%C3\%B3n_inicial_docente_en_Colomb ia

Rebolledo-Rebolledo, R. (2020). La investigación en la formación inicial docente de profesores de Historia y Geografía: Galimatías de fines desde los documentos curriculares. Revista de estudios y experiencias en educación, 19 (40), 111-128. http://dx.doi.org/10.21703/rexe.20201940rebolledo6

Rodríguez-de los Ríos, L. A. (2019). La investigación formativa: aspectos teóricos conceptuales. Revista Peruana de Psicología y Trabajo Social, 7 (2), 15-20. https://doi.org/10.32544/psicologia.v7i2.655

Ruano, L. E., Torres-Cap, A., \& Congote, E. (2017). Prácticas y discursos de la investigación formativa en Instituciones Educativas de nivel medio. Revista Atlas - Investigação 
$\begin{array}{llll}\text { Qualitativa em } & \text { Educação, } & 1 & \text { (1), }\end{array}$ https://proceedings.ciaiq.org/index.php/ciaiq2017/article/view/1563

Sabariego-Puig, M., Cano-Hila, A. B., Gros-Salvat, B., \& Piqué-Simón, B. (2020). Competencia investigadora e investigación formativa en la formación inicial del docente. Contextos Educativos. Revista de Educación, 1 (26), 239-259. https://doi.org/10.18172/con.4326

Salazar-Ayala, C. M., \& Gastélum-Cuadras, G. (2020). Teoría de la autodeterminación en el contexto de educación física: Una revisión sistemática. Revista Retos, 38 (38), 838-844. https://doi.org/10.47197/retos.v38i38.72729

Sánchez-Carlessi, H. H. (2017). La investigación formativa en la actividad curricular. Revista $\begin{array}{llllll}\text { Facultad } & \text { Medicina } & \text { Humana } & 17 & \text { (2), }\end{array}$ http://repositorio.urp.edu.pe/handle/urp/1060

Tapia-Sosa, E., Ledo-Royo, C. \& Estrabao-Pérez, A. E. (2017). Fundamentos epistemológicos de la investigación formativa en las universidades de ecuador.Didasc@ lia: Didáctica y Educación, 8 (7), 189-202. https://dialnet.unirioja.es/servlet/articulo?codigo=6694488 Turpo-Gebera, O., Mango-Quispe, P., Cuadros-Paz, L., \& Gonzales-Miñán, M. (2020). Formative research at the university: meanings conferred by faculty at an Education Department. Educação e Pesquisa, 46 (1), 1678-4634. https://doi.org/10.1590/s1678$\underline{4634202046215876}$

Turpo-Gebera, O., Gonzales-Miñán, M., Mango-Quispe, P., \& Cuadros-Paz, L. (2020). Sentidos docentes asignados a la enseñanza de la investigación formativa en una facultad de educación. Revista Propósitos y Representaciones, $8 \quad$ (3), 326-343. http://revistas.usil.edu.pe/index.php/pyr/article/view/326

Torres-Fonseca, A., \& López-Hernández, D. (2014). Criterios para publicar artículos de revisión sistemática. Revista de Especialidades Médico-Quirúrgicas, 19 (3), 393-399. https://www.redalyc.org/pdf/473/47332498021.pdf

Urbina-Cárdenas, J. E., Gamboa-Suárez, A. A., \& Prada-Núñez, R. (2020). Procesos formativos en investigación: relatos de estudiantes y profesores de un programa de trabajo $\begin{array}{llll}\text { social. Revista } & \text { Espacios, } & \text { (39), 22-31. }\end{array}$ https://www.revistaespacios.com/a20v41n39/a20v41n39p03.pdf

Ureña, Y. (2018). Actitudes frente a la investigación formativa y formación para la investigación en la Universidad Mariana Marianita Marroquín Y. En E. Serna (Ed). Revolución en la Formación y la Capacitación para el Siglo XXI. Editorial Instituto Antioqueño de Investigación. 
Velandia-Mesa, C., Serrano-Pastor, F. J., \& Martínez-Segura, M. J. (2017). Formative research in ubiquitous and virtual environments in higher education. Comunicar. Media Education Research Journal, 25 (1), 09-18. https://www.scipedia.com/public/VelandiaMesa_et_al_2017a

Velázquez, M. R., Amat-Abreu, M., Andrade-Santamaría, D. R., Jiménez-Martínez, R., \& Cisneros-Zúñiga, C. P. (2019). Development of formative research competences: challenges and perspectives for the university. Dilemas Contemporáneos: Educación, Política y Valore, 6 (1), 1- 26. https://www.proquest.com/docview/2245653337?pqorigsite $=$ gscholar $\&$ fromopenview $=$ true

Venegas-Mejía, V., Esquivel-Grados, J., \& Turpo-Gebera, O. (2019). Reflexiones sobre la investigación educativa y la investigación formativa en la Universidad Peruana. Conrado: revista pedagógica de la Universidad de Cienfuegos, 15 (70), 444454. $\quad$ http://scielo.sld.cu/scielo.php?script=sci_arttext\&pid=S1990$\underline{86442019000500444}$

Vera-Rojas, M. D. P., Gonzáles-Sánchez, E. C., Vera-Rojas, L. A., \& Chávez-Arias, S. (2018). La investigación formativa en la Universidad Ecuatoriana: una experiencia personal. Boletín Redipe, 7

(4), 89-98. https://dialnet.unirioja.es/servlet/articulo?codigo $=6523255$ 